\title{
Accelerating the Discovery of the beyond Rule of Five Compounds that have High Affinities toward SARS-CoV-2 Spike RBD
}

\author{
Abd Al-Aziz A. Abu-Saleh, ${ }^{*, \dagger}$ Arpita Yadav, ${ }^{\ddagger}$ and Raymond A. Poirier ${ }^{*, \dagger}$ \\ †Department of Chemistry, Memorial University, St. John's, NL A1B 3X', Canada \\ $\ddagger$ Department of Chemistry, University Institute of Engineering \& Technology, Chhatrapati \\ Shahu Ji Maharaj University, Kanpur 208024, India \\ E-mail: aabusaleh@mun.ca; rpoirier@mun.ca
}

\begin{abstract}
The battle against SARS-CoV-2 coronavirus is the focal point for the global pandemic that has affected millions of lives worldwide. The need for effective and selective therapeutics for the treatment of the disease caused by SARS-CoV-2 is critical. Herein, we performed computational de novo design incorporating molecular docking studies, molecular dynamics simulations, absolute binding energy calculations, and steered molecular dynamics simulations for the discovery of potential compounds with high affinity towards SARS-CoV-2 spike RBD. By leveraging ZINC15 database, a total of 1282 in-clinical and FDA approved drugs were filtered out from nearly 0.5 million protomers of relatively large compounds $(\mathrm{MW}>500$, and $\log \mathrm{P} \leq 5)$. Our results depict plausible mechanistic aspects related to the blockage of SARS-CoV-2 spike RBD by the top hits discovered. We found that the most promising candidates, namely, ZINC95628821, ZINC95617623, ZINC3979524, and ZINC261494658, strongly bind to
\end{abstract}


the spike RBD and interfere with the human ACE2 receptor. These findings accelerate the rational design of selective inhibitors targeting the spike RBD protein of SARS-CoV-2.

\section{Introduction}

The severe acute respiratory syndrome coronavirus $2(\mathrm{SARS}-\mathrm{CoV}-2)$ that emerged in late 2019 has caused serious illness and death all over the world. COVID-19, a disease caused by SARS-CoV-2, led the world health organization (WHO) to announce a global pandemic on March 11, 2020. To date, about 110 million infections and more than two million deaths have been reported by the WHO, and the numbers continue to rise. ${ }^{1}$ In late 2020 , among several vaccine candidates, two vaccines made by Moderna and Pfizer-BioNTech companies were approved by the U.S. food and drug administration (FDA) for an emergency use against COVID-19. Moreover, veklury (remdesivir), an antiviral drug, has also been approved by the U.S. FDA for the use as a treatment for COVID-19 in adults and pediatric patients (12 years of age and older) requiring hospitalization. Although studies reported that remdesivir failed to show clinical benefits for moderately severe COVID-19 patients, ${ }^{2,3}$ it is the only drug that has been approved so far for COVID-19 by the U.S. FDA. Therefore, accelerating the discovery of a safe and an effective COVID-19 medication is a must to control the pandemic.

The coronavirus genome encodes distinct structural and nonstructural proteins. The structural proteins (i.e., the membrane, the envelop, the spike, and the nucleocapsid) are responsible for key functions such as host infection, ${ }^{4}$ membrane fusion, ${ }^{5}$ self-assembly, ${ }^{6}$ release of virus-like particles, ${ }^{7}$ and other functions. ${ }^{8}$ The nonstructural proteins facilitate viral replication-transcription processes. ${ }^{9}$ The extensive amount of ongoing research is mainly focused on both non-structural and structural proteins of SARS-CoV-2 as drug targets in order to develop effective therapeutics for COVID-19. ${ }^{10-16}$ Among structural proteins, the spike protein is the key machinery that empowers virus entry into the host cell. ${ }^{17}$ Structural characterization of the spike protein would give atomic-level information to guide structure-based 
drug design. Recently, crystallographic and cryo-EM methods have been utilized to determine various structures and conformational states of the SARS-CoV-2 spike proteins. ${ }^{5,17,18}$ Amaro and coworkers have carried out massive molecular dynamics (MD) simulations to promote and extend the available structural data. ${ }^{19}$ Moreover, Choi et al performed MD simulations of the spike protein in a viral bilayer. ${ }^{20}$ These computational results added a detailed insight on the structural and dynamics of the full-length glycosylated SARS-CoV-2 spike protein at an atomic level. ${ }^{19}$

Detailed mechanisms of the cell entry of SARS-CoV-2 have been recently investigated. ${ }^{5,21,22}$ The spike protein has two subunits S1 and S2, which are critical for receptor recognition and membrane fusion, respectively. The $\mathrm{S} 1$ contains the receptor-binding domain (RBD) of the viral spike that specifically binds to the human angiotensin-converting enzyme 2 (hACE2). The RBD can be in either an up conformation, which is the receptor-accessible state (i.e., enables binding to the host receptor), or a down conformation, which is the receptor-inaccessible state. ${ }^{17,21}$ Gur et al investigated conformational dynamics and the transition pathway between down to up states of the spike protein by using all-atom MD simulations. ${ }^{23}$ For membrane fusion, host cell proteases cleave SARS-CoV-2 spikes at the S1/S2 boundary followed by structural changes of the S2 that promote a host cell entry. ${ }^{21,24,25}$ Interestingly, experimental results have revealed that SARS-CoV-2 RBD has stronger hACE2 binding affinity than that possessed by the previous SARS-CoV RBD. However, the entire SARS-CoV-2 spike has hACE2 binding affinity similar to or lower than SARS-CoV-2 spike. ${ }^{18,21,26,27}$ Moreover, a computational study of the RBD -accessible and -inaccessible conformations and their binding strength to the hACE2 is in agreement with the experimental findings. ${ }^{28}$ Therefore, the RBD is considered as a key target for designing and developing compounds that suppress the virus entry into the human cell.

Different approaches have been conducted that are based on interfering with the RBD binding to the hACE2 including neutralizing antibodies, ${ }^{29-31}$ decoy proteins, ${ }^{32}$ miniproteins, ${ }^{33}$ peptides, ${ }^{34-40}$ and stapled hACE2 peptides. ${ }^{41}$ In addition, many studies have ex- 
plored drug repurposing or repositioning of vast chemical compounds such as FDA approved drugs, ${ }^{42-44}$ antivirals, ${ }^{45,46}$ phytochemicals,${ }^{47}$ essential free fatty acids, ${ }^{48}$ and natural products $^{49,50}$ for the sake of disrupting the RBD-hACE2 binding. Designing compounds for inhibiting the hACE2 active site has also been reported. ${ }^{51}$

As far as we are aware, no high-throughput virtual screening has been conducted in relatively large molecules in the "beyond rule of 5 " chemical space that have high RBD binding affinity. Herein, we performed a comprehensive computational protocol, which incorporates molecular docking, MD simulations, absolute binding energy calculations, and steered MD simulations for the discovery of relatively large compounds that would bind to SARS-CoV-2 spike RBD very tightly, therefore, blocking viral attachment to the host cell.

\section{Computational Methods}

\section{Model System Preparation}

The initial structure of SARS-CoV-2 RBD bound to the hACE2 was retrieved from the RCSB protein data bank $[\mathrm{PDB}$ entry $6 \mathrm{LZG}] .{ }^{5}$ The protonation states of the titratable residues were determined using the $\mathrm{H}++$ Web server under the physiological $\mathrm{pH} .{ }^{52}$ Moreover, salinity, internal and external dielectric constants were set to be $0.15,10.0$, and 80.0 respectively.

\section{Molecular Docking}

3-D chemical structures $(\mathrm{MW}>500$, and $\operatorname{LogP} \leq 5)$ were retrieved from ZINC15 database, ${ }^{53}$ resulting in about 0.5 million protomers. Among these protomers, only in-clinical trials and FDA approved drugs (a total of 1283 protomers) were selected for docking. OpenBabel software $^{54}$ was used to minimize selected compounds. These compounds were docked into a preprocessed SARS-CoV-2 RBD protein pocket by utilizing the AutoDock Vina. ${ }^{55}$ For improving the reliability of docking, these compounds were also docked using the BindScope web application. ${ }^{56}$ The xyz grid cell origin of $-37.0 \AA, 30.0 \AA$, and $3.5 \AA$ with dimensions of 
$26.0,45.5$, and 22.0 in the $\mathrm{x}, \mathrm{y}$, and $\mathrm{z}$ directions, respectively, was built for docking calculations. The grid cell was constructed at key residues located within receptor binding motif of the RBD that are directly involved in interacting with the hACE2. The spike RBD-ligand binding affinities were ranked based on a consensus docking results from two scoring functions Vina ${ }^{55}$ and 3D-convolutional neural networks method (Kdeep). ${ }^{57}$ Consensus docking scores were chosen as the measure of protein-ligand binding affinities to rank the poses of the studied compounds. For the next high-throughput virtual screening, we selected the top 13 compounds that have a consensus docking score of $\leq-38 \mathrm{~kJ} \mathrm{~mol}^{-1}\left(-9 \mathrm{kcal} \mathrm{mol}^{-1}\right)$. Moreover, we selected one compound from each scoring functions Vina ${ }^{55}$ and Kdeep algorithm ${ }^{57}$ that has the highest protein-ligand binding affinity. All molecular docking results are provided in the supporting information (SI).

\section{Simulations}

All-atom MD simulations were done using the NAMD 2.13 package. ${ }^{58}$ We performed MD simulations on the model systems with top docking results (total of 15 RBD-ligand complexes). Model systems of the RBD-ligand complexes were prepared in an explicit solvent using the TIP3P water model ${ }^{59}$ in a simulation cell with dimensions of $86 \times 71 \times 96 \AA^{3}$. All crystallographically resolved water molecules were removed from model systems. The parameters for the ligands and the spike RBD protein model structures were all set using the CHARMM general force field (CGenFF), ${ }^{60}$ and the CHARMM36 force field, ${ }^{61}$ respectively. Model systems were neutralized using chloride ions. The MD protocols involve minimization, annealing, equilibration, and production. The protein backbone atoms were restrained in the minimization and annealing simulations. The $\mathrm{C} \alpha$ atoms of the protein backbone were restrained in the 1 ns equilibration simulations. For the 100 ns MD production simulations, no atoms were restrained. The isothermal-isobaric (NPT) ensemble and a 2 fs time-step was chosen for all MD simulations. The pressure was set at 1 atm using the Nosé-Hoover Langevin piston barostat ${ }^{62,63}$ with the Langevin piston decay of 0.2 ps and a period of 0.4 
ps. The temperature was set at $298.15 \mathrm{~K}$ using the Langevin thermostat ${ }^{64}$ and a damping frequency of $1 \mathrm{ps}^{-1}$. A cutoff distance of $10.0 \AA$ was applied for Lennard-Jones interactions with a pair list distance of $12 \AA$, interactions were smoothly truncated at $8.0 \AA$. The particle-mesh Ewald (PME) method wad utilized to treat the long-range electrostatic interactions ${ }^{65}$ where a grid spacing of $1.0 \AA$ was used for all simulation cells. Covalent bonds involving hydrogen atoms were constrained using the SHAKE algorithm. ${ }^{66}$ See the SI for more information about the MD simulations protocol.

\section{Absolute Binding Energy Calculations}

One of the main goal in computer-aided drug discovery is the accurate and reliable prediction of the absolute binding energy of receptor-ligand systems. Here, we calculated the absolute binding energies of the stable RBD-ligand complexes that resulted from the MD simulations. Starting from the equilibrated RBD-ligand structures, we conducted the absolute binding energy calculations using the potential of mean force (PMF) approach as described by Chipot and coworkers. ${ }^{67,68}$ It has been reported that the PMF approach accurately predicts protein-ligand binding affinities. ${ }^{67,69} \mathrm{~A}$ set of geometrical restraints on collective variables ${ }^{70}$ are exploited to accurately determine the translational, rotational, and conformational entropies that accompany the binding process and, thus, computing protein-ligand absolute binding energies. These restraints include the root mean-square deviation (RMSD) of the ligand in the bound (site) and unbound (bulk) states compared to an equilibrated reference structure of the RBD-ligand complex. Additional restraints include three Euler $(\Theta, \Phi, \Psi)$ and two spherical $(\theta, \varphi)$ angles that describe the relative orientation and position of the ligand with respect to the RBD protein, respectively, see Figure S4 in the SI. The equilibrium binding constant $\left(K_{e q}\right)$ can be determined as,

$$
K_{\text {eq }}=\exp \left[-\beta\left(\Delta G_{c}^{\text {site }}+\Delta G_{c}^{\text {bulk }}+\Delta G_{o}^{\text {site }}+\Delta G_{a}^{\text {site }}+\Delta G_{o}^{\text {bulk }}-\frac{1}{\beta} \ln \left(S^{*} I^{*} C^{\circ}\right)\right)\right]
$$


where the $\Delta G_{c}^{\text {site }}$ and $\Delta G_{c}^{b u l k}$ denote the Gibbs energy changes of the conformational ligand RMSD in the site and bulk states, respectively, the $\Delta G_{o}^{\text {site }}$ and $\Delta G_{a}^{\text {site }}$ represent the Gibbs energy changes associated with the orientational and the positional of the ligand in the binding site, respectively, and the $\Delta G_{o}^{b u l k}$ represents the Gibbs energy change associated with the ligand orientation in the bulk. The last term in Eq. (1) represents the separation of the ligand from the binding site into the bulk. It should be mentioned that the $\Delta G_{o}^{b u l k}$ is calculated analytically as reported by Chipot and coworkers. ${ }^{67}$ More details on theoretical background of the binding energy calculations using the PMF route can be obtained elsewhere. ${ }^{67}$ Eventually, the standard binding energy is

$$
\Delta G_{b i n d}^{\circ}=k_{B} T \ln K_{e q} C^{\circ}
$$

where the $k_{B}$ and $T$ are the Boltzmann constant and simulation temperature, respectively, and $C^{\circ}=1 / 1661 \AA^{3}$ is the standard one molar concentration. For each model system, the RBD-ligand separation, RMSD (site), RMSD (bulk), and angular course variables were run for 50 ns, 50 ns, 100 ns, and 50 ns, respectively. By leveraging GPU-accelerated NAMD

engine, ${ }^{71}$ the simulations were performed in triplicate, yielding an aggregate total of $750 \mathrm{~ns}$ simulation time for each model system. Absolute binding energies reported in this study are the averages of the triplicate runs. See the SI for more details about the absolute binding energy protocol.

\section{Steered Molecular Dynamics (SMD) Simulations}

To probe the selectivity and the potency of the top hits binding to the spike RBD protein, SMD simulations of the RBD-ligand-hACE2 model systems were conducted. The RBD-ligand-hACE2 model systems were solvated using a TIP3P water model with a $12 \AA$ buffer around the system and then extended $30.0 \AA$ in the SMD pulling axis, resulting in a simulation cell with dimensions of $121 \times 121 \times 167 \AA^{3}$. The SMD simulations were employed 
by harmonically restraining the position of the spike RBD protein center-of-mass, and moving the second restraint center-of-mass of the hACE2 protein, with a constant pulling speed of $1.5 \AA \mathrm{ns}^{-1}$ in the $\mathrm{z}$-axis direction. More details about the SMD protocol are provided in the SI.

\section{Results and discussion}

We performed an integrated computational strategy including molecular docking, MD simulations, absolute binding energy calculations, and SMD simulations to accelerate the identification of promising drug candidates for COVID-19. 1283 compounds (in-clinical trials and FDA approved drugs) were retrieved from the ZINC15 database ${ }^{53}$ and advanced to flexible molecular docking using both AutoDock Vina ${ }^{55}$ and Kdeep ${ }^{57}$ scoring functions. Consensus docking scores were chosen as an initial measure of the RBD-ligand binding affinities to rank the poses of the screened compounds. Docking results of the top 15 compounds are listed in Table 1. The two-dimensional structures and the three-dimensional docked conformations of these compounds are also provided in the SI (Figures S1 and S2, respectively). Docking results of all screened compounds are provided in the SI.

The best 15 RBD-ligand complexes from molecular docking were used as starting structures for the conventional MD simulations. Ensemble MD simulations consolidate the effect of solvation, flexibility of both ligand and protein target, and better estimate of proteinligand nonbonded interactions. The structure stability of the RBD-ligand model systems were evaluated by the RMSD with respect to the equilibrated structures (see Figure S3 in the SI). The time-evolution of the RMSD show that most ligands with invalid binding modes reveal significant signs of binding instability in the RBD binding pocket. Snapshots of the last frame of the stable simulated RBD-ligand model systems are depicted in Figure 1.

The use of absolute binding energy calculations is crucial to enhance the hit identification. Consequently, rigorous absolute binding energy calculations were only conducted on the 


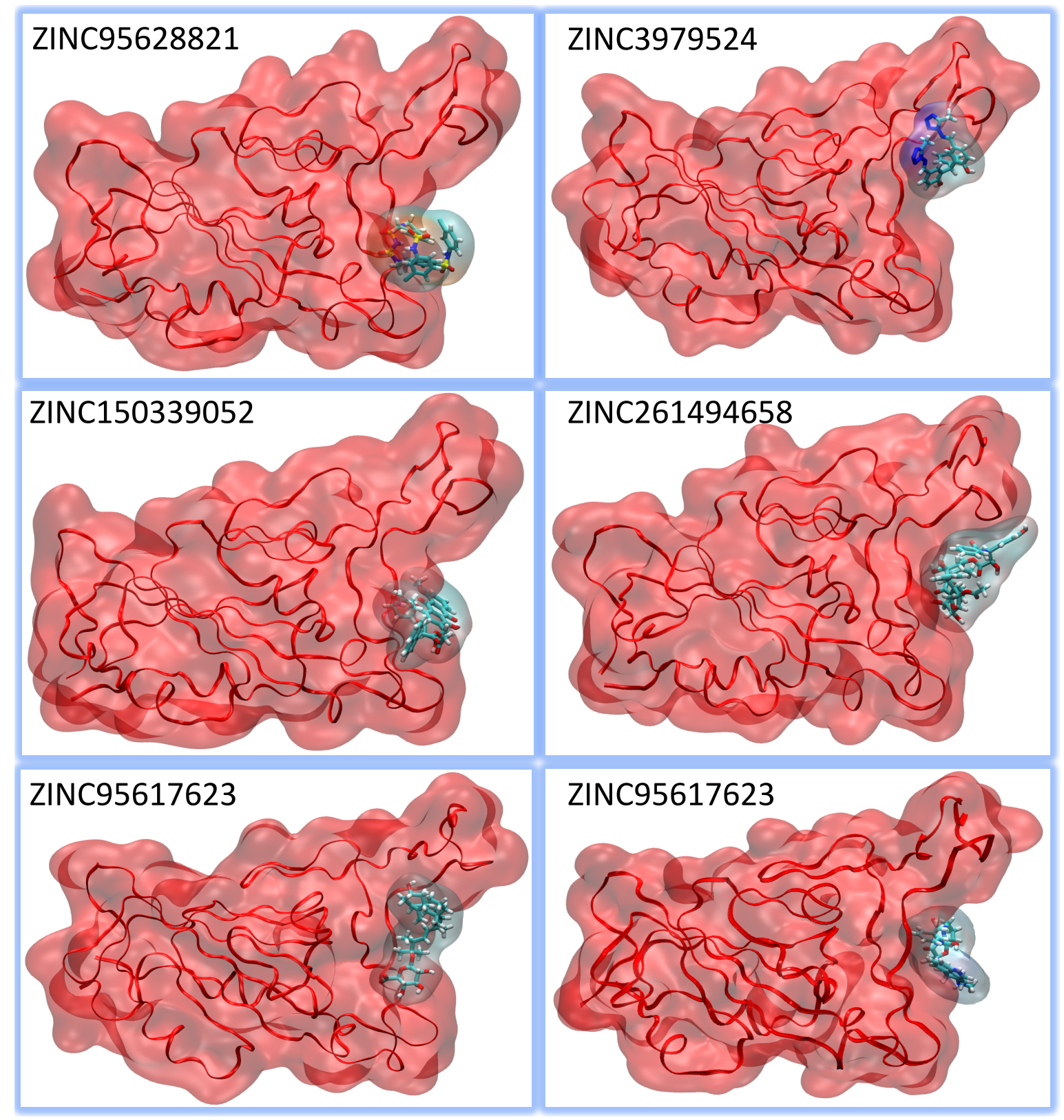

Figure 1: The last frame of the $100 \mathrm{~ns}$ MD simulations of ligands complexed with the spike $\mathrm{RBD}$ protein. ligands are shown as sticks and the RBD is shown as red ribbons. 
Table 1: Summary of top consensus docking results in $\mathrm{kJ} \mathrm{mol}^{-1}$ for SARS-CoV-2 spike RBD target

\begin{tabular}{llll}
\hline Compound ID & Vina score & Kdeep score & Consensus score \\
\hline ZINC6717782 & -38 & -40 & -39 \\
ZINC95628821 & -36 & -41 & -39 \\
ZINC253638647 & -35 & -43 & -38 \\
ZINC3979524 & -36 & -41 & -38 \\
ZINC253387884 & -35 & -41 & -38 \\
ZINC255977094 & -36 & -40 & -38 \\
ZINC150339052 & -37 & -39 & -38 \\
ZINC261494659 & -35 & -41 & -38 \\
ZINC936069565 & -34 & -42 & -38 \\
ZINC51951669 & -37 & -38 & -38 \\
ZINC49637509 & -32 & -43 & -38 \\
ZINC261494658 & -33 & -42 & -38 \\
ZINC95617623 $_{\text {ZINC261494590 }}^{a}$ & -32 & -43 & -38 \\
ZINC3978005 $^{b}$ & -40 & -47 & -36 \\
\hline
\end{tabular}

${ }^{a}$ The compound was selected based on the Kdeep scoring function; ${ }^{b}$ The compound was selected based on the Vina scoring function.

stable RBD-ligand systems resulting form the MD simulations. In absolute binding energy calculations, the resulting contributions from the aforementioned restraints are computed sequentially using the extended adaptive biasing force (eABF) method ${ }^{72}$ with the unbiased corrected z-averaged restraint (CZAR) estimator ${ }^{73}$ implemented in the Colvars module ${ }^{70}$ of NAMD. The contributions from the course variables and the final standard binding energy $\left(\Delta G_{\text {bind }}^{\circ}\right)$ of ligands to the SARS-CoV-2 spike RBD protein are provided in Table 2.

Table 2: The calculated contributions accompanying the RBD-ligand binding process and the predicted $\Delta G_{b i n d}^{\circ}$ in $\mathrm{kJ} \mathrm{mol}^{-1}$

\begin{tabular}{llllll}
\hline Protein-ligand complex & $\Delta G_{c}^{\text {site }}$ & $\Delta G_{\text {angular }}^{\text {site }}$ & $-\frac{1}{\beta} \ln \left(S^{*} I^{*} C^{\circ}\right)$ & $\Delta G_{c}^{\text {bulk }}$ & $\Delta G_{\text {bind }}^{\circ}$ \\
\hline RBD-ZINC95628821 & $-73 \pm 6$ & $-5 \pm 0$ & $-44 \pm 6$ & $72 \pm 3$ & $-23 \pm 3$ \\
RBD-ZINC3979524 & $-22 \pm 4$ & $-5 \pm 0$ & $-38 \pm 2$ & $22 \pm 2$ & $-12 \pm 3$ \\
RBD-ZINC150339052 & $-41 \pm 5$ & $-9 \pm 3$ & $-12 \pm 3$ & $38 \pm 3$ & $+3 \pm 2$ \\
RBD-ZINC261494658 & $-33 \pm 2$ & $-7 \pm 0$ & $-24 \pm 3$ & $29 \pm 1$ & $-8 \pm 2$ \\
RBD-ZINC95617623 & $-38 \pm 4$ & $-9 \pm 1$ & $-26 \pm 4$ & $27 \pm 1$ & $-19 \pm 1$ \\
RBD-ZINC3978005 & $-21 \pm 5$ & $-9 \pm 1$ & $-12 \pm 1$ & $19 \pm 3$ & $+4 \pm 4$ \\
\hline
\end{tabular}

Unambiguously, the four ligands, namely, ZINC95628821, ZINC95617623, ZINC3979524, 
and ZINC261494658 bind spontaneously to the SARS-CoV-2 spike RBD with $\Delta G_{\text {bind }}^{\circ}$ of $-23,-19,-12$, and -8 , respectively. In contrast, the two ligands, namely, ZINC150339052 and ZINC3978005 have non-spontaneous binding behavior toward the RBD with $\Delta G_{\text {bind }}^{\circ}=$ 3 and $4 \mathrm{~kJ} \mathrm{~mol}^{-1}$, respectively. These values represent the non-covalent interaction energies between the ligands and the spike RBD and give a good estimate of their binding affinities.

To validate the potency of the RBD-ligand binding affinities, we need to investigate if the top hits are able to interfere with the RBD-hACE2 binding event. For this purpose, SMD simulations were employed for distinguishing active from decoy ligands. Both strong and weak ligands listed in Table 2 were considered for SMD simulations as a validation strategy. In SMD simulations, the center of mass of the RBD was kept fixed (i.e., PHE400 of the $\mathrm{RBD}$ is an anchoring residue), whereas the center of mass of the hACE2 was steered (i.e., TYR515 of the hACE2 is a pulling residue). Figure 2 shows the final results of 20 ns SMD simulations for the RBD-hACE2 in the presence and absence of ligands.

The initial and the equilibrated conformations of RBD-ligand-hACE2 model systems are also provided in the SI, Figure S5. The time-evolution force profile of the unbinding event of the RBD-ACE2 model system has a maximum rupture force of $704 \mathrm{pN}$, in a good agreement with the computed maximum rupture force of $751 \mathrm{pN}$ reported recently. ${ }^{74}$ Taka et al also reported the unbinding process of SARS-CoV-2 spike RBD from the hACE2 by carrying out SMD simulations. ${ }^{75}$ It is well established that the rupture force is directly proportional with the receptor-ligand binding affinity. ${ }^{76}$ The moving average (black line) of the time-evolution force profiles in Figure 2 indicates that ligands (particularly ZINC95628821, ZINC3979524, and ZINC95617623) are disrupting the RBD-hACE2 binding by lowering the rupture force. Additionally, the structures in Figure 2b show that ligands, namely, ZINC95628821, ZINC261494658, and ZINC95617623 are strong binders to the RBD protein. This is anticipated due to the fact that these ligands have relatively better binding affinity to the RBD than other ligands, namely, ZINC150339052, and ZINC3978005. On the contrary, during the SMD simulations, ZINC3979524 and ZINC3978005 left the RBD and attached 

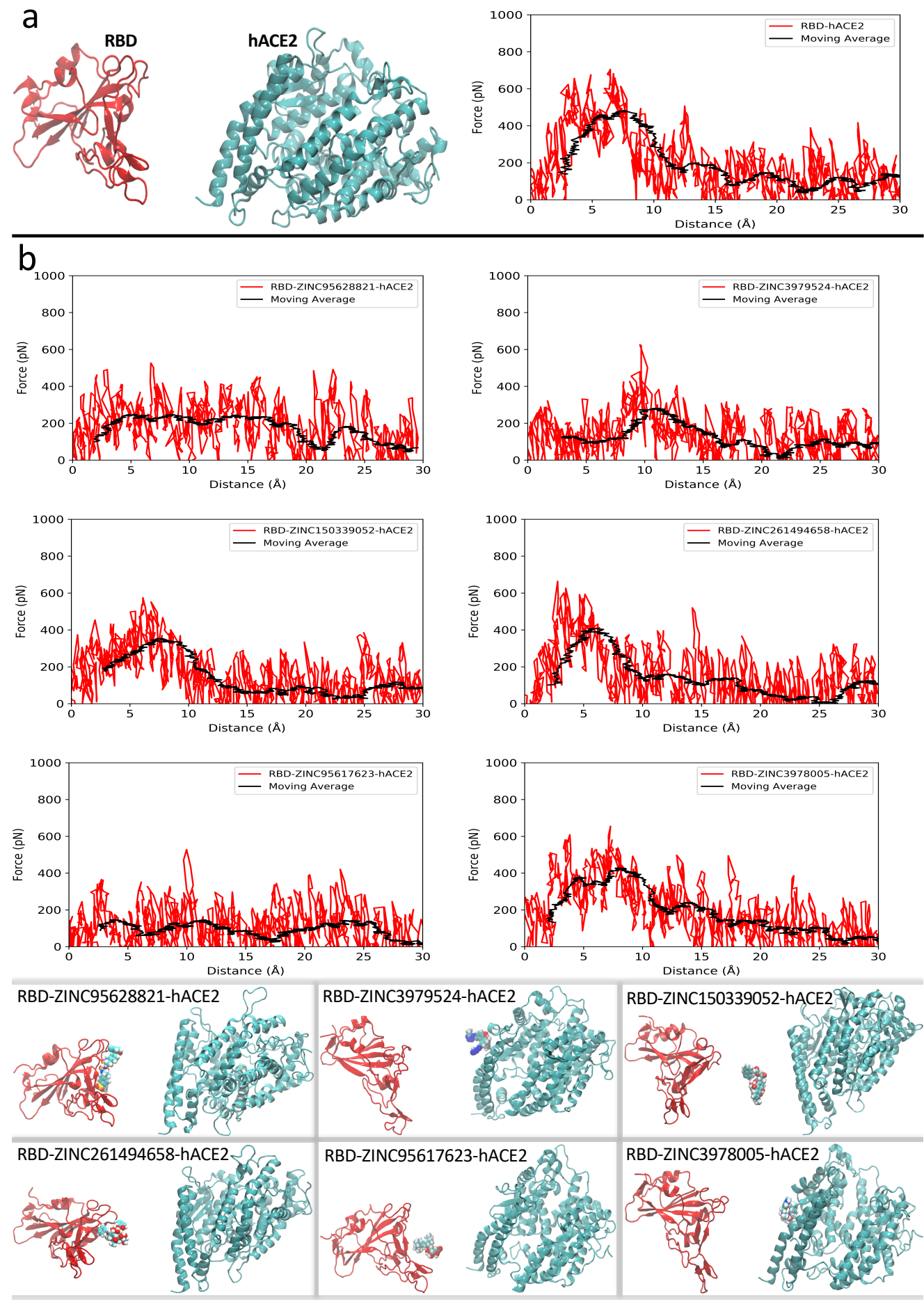

Figure 2: (a) The structure of the apo RBD-hACE2 (left) along with its time-averaged SMD results (right); (b) the structures of the RBD-ligand-hACE2 (bottom) along with their timeaveraged SMD results (top). All structures were depicted after the 20 ns SMD simulations. The RBD, the hACE2, and the ligands are shown as red cartoon, cyan cartoon, spheres, respectively. 
to the hACE2 receptor, revealing that these ligands have more favourable interactions to the hACE2 than the RBD. SMD simulations also revealed that ZINC150339052 has non promising binding affinity toward neither the RBD nor the hACE2 proteins (see Figure $2 \mathrm{~b}$ ).

Detailed analyses of the ligand interaction diagram of the equilibrated RBD-ligand-hACE2 complexes are shown in Figure 3. Ligands that exhibit robust RBD binding stability during the SMD simulations, namely, ZINC95628821, ZINC261494658, and ZINC95617623, indeed form more non-covalent interactions (i.e. hydrogen bonding, pi-cation, pi-pi stacking) with key amino acid residues of the RBD and enjoy more exposure to the RBD binding pose than other ligands. Notably, these robust binders have direct polar contact with ARG403 of the RBD. Moreover, these ligands also interfere with key residues of the hACE2 protein (i.e. ASP38, TYR41, and LYS353). Interestingly, ARG403 of the RBD and residues ASP38, TYR41, and LYS353 of the hACE2 were reported as true hot spots that contribute to the stability of the RBD-hACE2 interface. ${ }^{77}$ Many studies reported that hydrophobic interactions also play a critical role in anchoring the RBD to the hACE2 receptor. ${ }^{75,78}$ All ligands that possess spontaneous standard binding energies have at least four direct contacts with the RBD hydrophobic residues (see Figure 3). The polar and non-polar interactions of the RBD-ligand complexes contribute to their total standard binding energy. Based on rigorous binding energy calculations and SMD simulations, we suggest that the top drug candidates are ZINC95628821 followed by ZINC95617623, ZINC3979524, and ZINC261494658. These top hits certify critical interactions associated with the RBD-ligand-hACE2 binding, which in turn disrupt the RBD-hACE2 binding event.

\section{Conclusions}

In this work, we performed high-throughput virtual screening that comprise of molecular docking, MD simulations, non-covalent absolute binding energy calculations, and SMD simulations for the discovery of SARS-CoV-2 spike RBD-based inhibitors. Vina ${ }^{55}$ and 


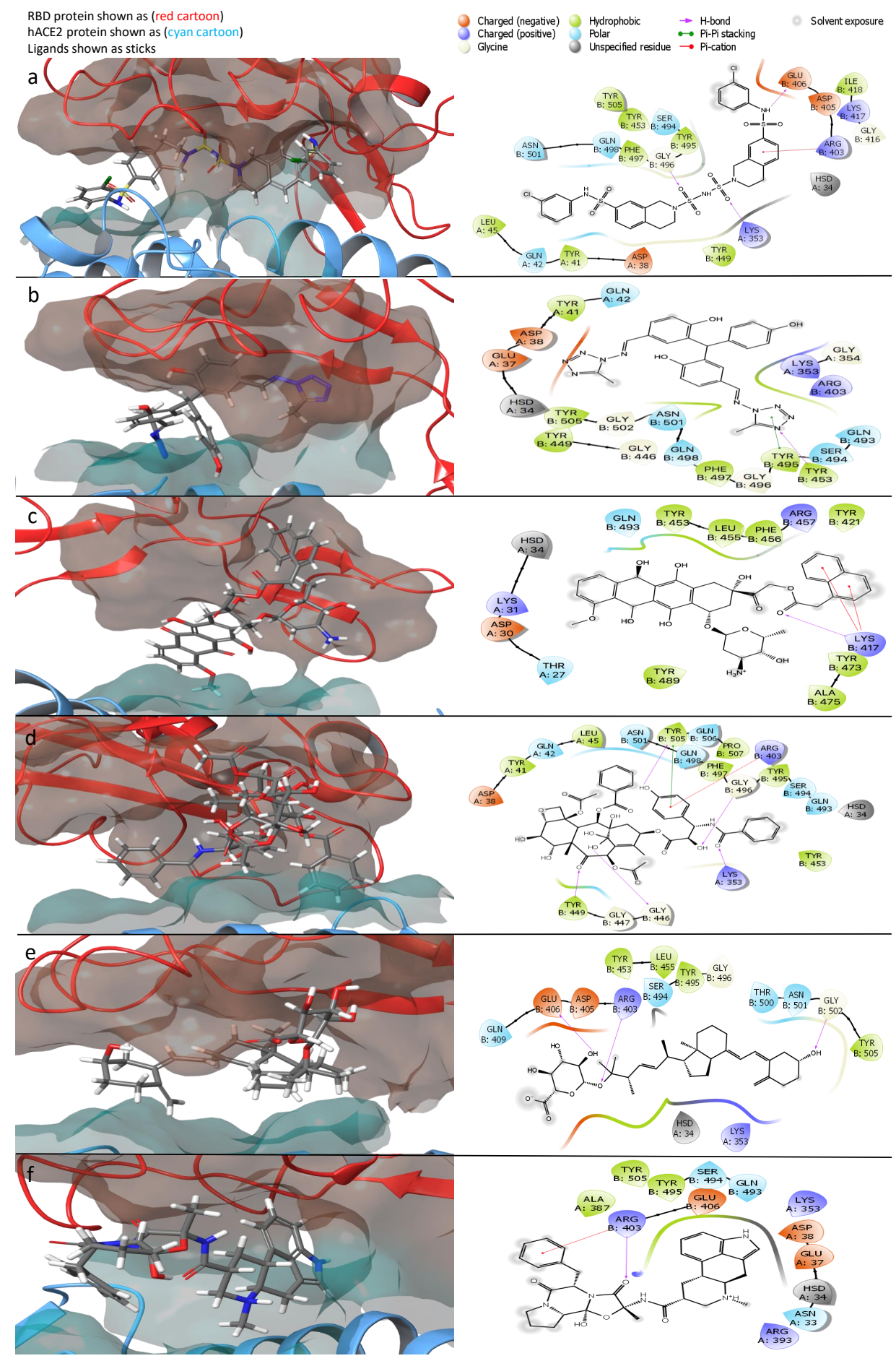

Figure 3: Representative poses (left) detailed ligand interaction diagrams (right) of the RBD-ligand-ACE2 complexes. (a) ZINC95628821, (b) ZINC3979524, (c) ZINC150339052, (d) ZINC261494658, (e) ZINC95617623, (f) ZINC3978005. In the ligand interaction diagram, the hACE2 and the RBD residues were labeled A and B, respectively. 
Kdeep ${ }^{57}$ scoring functions were leveraged for structure-based screening and ranking of inclinical trials and FDA approved drugs. Absolute binding energy calculations in combinations with the SMD simulations provided more effective ranking procedures of the top hits. Based on integrated in silico approach, our findings suggest that ZINC95628821, ZINC95617623, ZINC3979524, and ZINC261494658 have potential antiviral effects by blocking the RBD-hACE2 recognition. Clinical trials have shown that ZINC3979524 is a novel inhibitor of a respiratory syncytial virus with the EC50 of $1.4 \mathrm{nM}$, and thus, this compound along with the other drug candidates suggested herein need to be considered for further in vitro and in vivo investigation. This study provides a rational drug design of RBD-based inhibitors for accelerating the research and development of effective COVID-19 medications.

\section{Acknowledgement}

The authors thank Compute Canada for computer time. R.A.P. is grateful to the Natural Sciences and Engineering Council of Canada (NSERC) for financial support. A.A.A thanks the School of Graduate Studies of Memorial University for funding. A.Y. thanks Science and Engineering Research Board, New Delhi for research grant number EMR/2016/000769. A.Y is also grateful to Shastri Indo Canadian Institute, New Delhi for Shastri Scholar Travel Subsidy Grant in the year 2016.

\section{Supporting Information Available}

Results of molecular docking, protocols and parameters used for MD simulations, absolute binding energy calculations, and SMD simulations.

\section{Notes}

The authors declare no competing financial interest. 


\section{References}

(1) COVID-19 weekly epidemiological update; World Health Organization, 23 February 2021.

(2) Wang, Y.; Zhang, D.; Du, G.; Du, R.; Zhao, J.; Jin, Y.; Fu, S.; Gao, L.; Cheng, Z.; Lu, Q., et al. Remdesivir in adults with severe COVID-19: a randomised, double-blind, placebo-controlled, multicentre trial. The Lancet 2020, 395, 1569-1578.

(3) Young, B.; Tan, T. T.; Leo, Y. S. The place for remdesivir in COVID-19 treatment. The Lancet. Infectious Diseases 2021, 21, 20.

(4) Hossain, M. G.; Akter, S.; Saha, S. SARS-CoV-2 host diversity: An update of natural infections and experimental evidences. Journal of Microbiology, Immunology and Infection 2020,

(5) Wang, Q.; Zhang, Y.; Wu, L.; Niu, S.; Song, C.; Zhang, Z.; Lu, G.; Qiao, C.; Hu, Y.; Yuen, K.-Y., et al. Structural and functional basis of SARS-CoV-2 entry by using human ACE2. Cell 2020, 181, 894-904.

(6) Ye, Q.; West, A. M.; Silletti, S.; Corbett, K. D. Architecture and self-assembly of the SARS-CoV-2 nucleocapsid protein. Protein Science 2020, 29, 1890-1901.

(7) Siu, Y.; Teoh, K.; Lo, J.; Chan, C.; Kien, F.; Escriou, N.; Tsao, S.; Nicholls, J.; Altmeyer, R.; Peiris, J., et al. The M, E, and N structural proteins of the severe acute respiratory syndrome coronavirus are required for efficient assembly, trafficking, and release of virus-like particles. Journal of virology 2008, 82, 11318-11330.

(8) Mittal, A.; Manjunath, K.; Ranjan, R. K.; Kaushik, S.; Kumar, S.; Verma, V. COVID19 pandemic: Insights into structure, function, and hACE2 receptor recognition by SARS-CoV-2. PLoS pathogens 2020, 16, e1008762. 
(9) Haque, S. M.; Ashwaq, O.; Sarief, A.; Azad John Mohamed, A. K. A comprehensive review about SARS-CoV-2. Future virology 2020, 15, 625-648.

(10) Izda, V.; Jeffries, M. A.; Sawalha, A. H. COVID-19: A review of therapeutic strategies and vaccine candidates. Clinical Immunology 2020, 108634.

(11) Liu, X.-H.; Zhang, X.; Lu, Z.-H.; Zhu, Y. S.; Wang, T. Potential molecular targets of nonstructural proteins for the development of antiviral drugs against SARS-CoV-2 infection. Biomedicine $\&$ Pharmacotherapy 2020, 111035.

(12) Mulholland, A. J.; Amaro, R. E. COVID19-Computational chemists meet the moment. 2020, 60, 5724-5726.

(13) Li, Z.; Li, X.; Huang, Y.-Y.; Wu, Y.; Liu, R.; Zhou, L.; Lin, Y.; Wu, D.; Zhang, L.; Liu, H., et al. Identify potent SARS-CoV-2 main protease inhibitors via accelerated free energy perturbation-based virtual screening of existing drugs. Proceedings of the National Academy of Sciences 2020, 117, 27381-27387.

(14) Abu-Saleh, A. A.-A. A.; Awad, I. E.; Yadav, A.; Poirier, R. A. Discovery of potent inhibitors for SARS-CoV-2's main protease by ligand-based/structure-based virtual screening, MD simulations, and binding energy calculations. Physical Chemistry Chemical Physics 2020, 22, 23099-23106.

(15) Awoonor-Williams, E.; Abu-Saleh, A. A.-A. A. Covalent and non-covalent binding free energy calculations for peptidomimetic inhibitors of SARS-CoV-2 main protease. Physical Chemistry Chemical Physics 2021, 23, 6746-6757.

(16) Zhang, H.; Yang, Y.; Li, J.; Wang, M.; Saravanan, K. M.; Wei, J.; Tze-Yang Ng, J.; Tofazzal Hossain, M.; Liu, M.; Zhang, H., et al. A novel virtual screening procedure identifies Pralatrexate as inhibitor of SARS-CoV-2 RdRp and it reduces viral replication in vitro. PLoS computational biology 2020, 16, e1008489. 
(17) Wrapp, D.; Wang, N.; Corbett, K. S.; Goldsmith, J. A.; Hsieh, C.-L.; Abiona, O.; Graham, B. S.; McLellan, J. S. Cryo-EM structure of the 2019-nCoV spike in the prefusion conformation. Science 2020, 367, 1260-1263.

(18) Walls, A. C.; Park, Y.-J.; Tortorici, M. A.; Wall, A.; McGuire, A. T.; Veesler, D. Structure, function, and antigenicity of the SARS-CoV-2 spike glycoprotein. Cell 2020, 181, 281-292.

(19) Casalino, L.; Gaieb, Z.; Goldsmith, J. A.; Hjorth, C. K.; Dommer, A. C.; Harbison, A. M.; Fogarty, C. A.; Barros, E. P.; Taylor, B. C.; McLellan, J. S., et al. Beyond shielding: the roles of glycans in the SARS-CoV-2 spike protein. ACS Central Science 2020, 6, 1722-1734.

(20) Choi, Y. K.; Cao, Y.; Frank, M.; Woo, H.; Park, S.-J.; Yeom, M. S.; Croll, T. I.; Seok, C.; Im, W. Structure, Dynamics, Receptor Binding, and Antibody Binding of the Fully Glycosylated Full-Length SARS-CoV-2 Spike Protein in a Viral Membrane. Journal of Chemical Theory and Computation 2021, 17, 2479-2487.

(21) Shang, J.; Wan, Y.; Luo, C.; Ye, G.; Geng, Q.; Auerbach, A.; Li, F. Cell entry mechanisms of SARS-CoV-2. Proceedings of the National Academy of Sciences 2020, 117, $11727-11734$.

(22) Tang, T.; Jaimes, J. A.; Bidon, M. K.; Straus, M. R.; Daniel, S.; Whittaker, G. R. Proteolytic activation of SARS-CoV-2 spike at the S1/S2 boundary: potential role of proteases beyond furin. ACS Infectious Diseases 2021, 7, 264-272.

(23) Gur, M.; Taka, E.; Yilmaz, S. Z.; Kilinc, C.; Aktas, U.; Golcuk, M. Conformational transition of SARS-CoV-2 spike glycoprotein between its closed and open states. The Journal of Chemical Physics 2020, 153, 075101.

(24) Letko, M.; Marzi, A.; Munster, V. Functional assessment of cell entry and receptor 
usage for SARS-CoV-2 and other lineage B betacoronaviruses. Nature microbiology 2020, 5, 562-569.

(25) Borkotoky, S.; Dey, D.; Banerjee, M. Computational insight into the mechanism of SARS-CoV-2 membrane fusion. Journal of Chemical Information and Modeling 2021, $61,423-431$.

(26) Ou, X.; Liu, Y.; Lei, X.; Li, P.; Mi, D.; Ren, L.; Guo, L.; Guo, R.; Chen, T.; Hu, J., et al. Characterization of spike glycoprotein of SARS-CoV-2 on virus entry and its immune cross-reactivity with SARS-CoV. Nature communications 2020, 11, 1-12.

(27) Shang, J.; Ye, G.; Shi, K.; Wan, Y.; Luo, C.; Aihara, H.; Geng, Q.; Auerbach, A.; Li, F. Structural basis of receptor recognition by SARS-CoV-2. Nature 2020, 581, 221-224.

(28) Peng, C.; Zhu, Z.; Shi, Y.; Wang, X.; Mu, K.; Yang, Y.; Zhang, X.; Xu, Z.; Zhu, W. Computational Insights into the Conformational Accessibility and Binding Strength of SARS-CoV-2 Spike Protein to Human Angiotensin-Converting Enzyme 2. The journal of physical chemistry letters 2020, 11, 10482-10488.

(29) Piccoli, L.; Park, Y.-J.; Tortorici, M. A.; Czudnochowski, N.; Walls, A. C.; Beltramello, M.; Silacci-Fregni, C.; Pinto, D.; Rosen, L. E.; Bowen, J. E., et al. Mapping neutralizing and immunodominant sites on the SARS-CoV-2 spike receptor-binding domain by structure-guided high-resolution serology. Cell 2020, 183, 1024-1042.

(30) Huo, J.; Zhao, Y.; Ren, J.; Zhou, D.; Duyvesteyn, H. M.; Ginn, H. M.; Carrique, L.; Malinauskas, T.; Ruza, R. R.; Shah, P. N., et al. Neutralization of SARS-CoV-2 by destruction of the prefusion spike. Cell host $\&$ microbe 2020, 28, 445-454.

(31) Wu, N. C.; Yuan, M.; Liu, H.; Lee, C.-C. D.; Zhu, X.; Bangaru, S.; Torres, J. L.; Caniels, T. G.; Brouwer, P. J.; Van Gils, M. J., et al. An alternative binding mode of IGHV3-53 antibodies to the SARS-CoV-2 receptor binding domain. Cell reports 2020, 33, 108274. 
(32) Linsky, T. W.; Vergara, R.; Codina, N.; Nelson, J. W.; Walker, M. J.; Su, W.; Barnes, C. O.; Hsiang, T.-Y.; Esser-Nobis, K.; Yu, K., et al. De novo design of potent and resilient hACE2 decoys to neutralize SARS-CoV-2. Science 2020, 370, 1208-1214.

(33) Cao, L.; Goreshnik, I.; Coventry, B.; Case, J. B.; Miller, L.; Kozodoy, L.; Chen, R. E.; Carter, L.; Walls, A. C.; Park, Y.-J., et al. De novo design of picomolar SARS-CoV-2 miniprotein inhibitors. Science 2020, 370, 426-431.

(34) Schütz, D.; Ruiz-Blanco, Y. B.; Münch, J.; Kirchhoff, F.; Sanchez-Garcia, E.; Müller, J. A. Peptide and peptide-based inhibitors of SARS-CoV-2 entry. Advanced drug delivery reviews 2020, 167, 47-65.

(35) Pomplun, S.; Jbara, M.; Quartararo, A. J.; Zhang, G.; Brown, J. S.; Lee, Y.-C.; Ye, X.; Hanna, S.; Pentelute, B. L. De novo discovery of high-affinity peptide binders for the SARS-CoV-2 spike protein. ACS Central Science 2021, 7, 156-163.

(36) Wang, G.; Yang, M.-L.; Duan, Z.-L.; Liu, F.-L.; Jin, L.; Long, C.-B.; Zhang, M.; Tang, X.-P.; Xu, L.; Li, Y.-C., et al. Dalbavancin binds ACE2 to block its interaction with SARS-CoV-2 spike protein and is effective in inhibiting SARS-CoV-2 infection in animal models. Cell research 2021, 31, 17-24.

(37) Chowdhury, S. M.; Talukder, S. A.; Khan, A. M.; Afrin, N.; Ali, M. A.; Islam, R.; Parves, R.; Al Mamun, A.; Sufian, M. A.; Hossain, M. N., et al. Antiviral peptides as promising therapeutics against SARS-CoV-2. The Journal of Physical Chemistry B 2020, 124, 9785-9792.

(38) Sitthiyotha, T.; Chunsrivirot, S. Computational Design of 25-mer Peptide Binders of SARS-CoV-2. The Journal of Physical Chemistry B 2020, 124, 10930-10942.

(39) Han, Y.; Král, P. Computational design of ACE2-based peptide inhibitors of SARSCoV-2. ACS nano 2020, 14, 5143-5147. 
(40) Freitas, F. C.; Ferreira, P. H. B.; Favaro, D. C.; Oliveira, R. J. d. Shedding Light on the Inhibitory Mechanisms of SARS-CoV-1/CoV-2 Spike Proteins by ACE2-Designed Peptides. Journal of chemical information and modeling 2021, 61, 1226-1243.

(41) Maas, M. N.; Hintzen, J. C.; Löffler, P. M.; Mecinović, J. Targeting SARS-CoV-2 spike protein by stapled hACE2 peptides. Chemical Communications 2021, 57, 3283-3286.

(42) Trezza, A.; Iovinelli, D.; Santucci, A.; Prischi, F.; Spiga, O. An integrated drug repurposing strategy for the rapid identification of potential SARS-CoV-2 viral inhibitors. Scientific reports 2020, 10, 1-8.

(43) Deganutti, G.; Prischi, F.; Reynolds, C. A. Supervised molecular dynamics for exploring the druggability of the SARS-CoV-2 spike protein. Journal of computer-aided molecular design 2021, 35, 195-207.

(44) Awad, I. E.; Abu-Saleh, A. A.-A. A.; Sharma, S.; Yadav, A.; Poirier, R. A. Highthroughput virtual screening of drug databanks for potential inhibitors of SARS-CoV-2 spike glycoprotein. Journal of Biomolecular Structure and Dynamics 2020, 1-14.

(45) Wang, X.; Cao, R.; Zhang, H.; Liu, J.; Xu, M.; Hu, H.; Li, Y.; Zhao, L.; Li, W.; Sun, X., et al. The anti-influenza virus drug, arbidol is an efficient inhibitor of SARS-CoV-2 in vitro. Cell discovery 2020, 6, 1-5.

(46) Puhl, A. C. et al. Repurposing the Ebola and Marburg Virus Inhibitors Tilorone, Quinacrine, and Pyronaridine: In Vitro Activity against SARS-CoV-2 and Potential Mechanisms. ACS Omega 2021, 6, 7454-7468.

(47) Pandey, P.; Rane, J. S.; Chatterjee, A.; Kumar, A.; Khan, R.; Prakash, A.; Ray, S. Targeting SARS-CoV-2 spike protein of COVID-19 with naturally occurring phytochemicals: an in silico study for drug development. Journal of Biomolecular Structure and Dynamics 2020, 1-11. 
(48) Toelzer, C.; Gupta, K.; Yadav, S. K.; Borucu, U.; Davidson, A. D.; Williamson, M. K.; Shoemark, D. K.; Garzoni, F.; Staufer, O.; Milligan, R., et al. Free fatty acid binding pocket in the locked structure of SARS-CoV-2 spike protein. Science 2020, 370, 725730.

(49) Li, Y.-T.; Yang, C.; Wu, Y.; Lv, J.-J.; Feng, X.; Tian, X.; Zhou, Z.; Pan, X.; Liu, S.; Tian, L.-W. Axial Chiral Binaphthoquinone and Perylenequinones from the Stromata of Hypocrella bambusae Are SARS-CoV-2 Entry Inhibitors. Journal of Natural Products 2021, 84, 436-443.

(50) Gangadevi, S.; Badavath, V. N.; Thakur, A.; Yin, N.; De Jonghe, S.; Acevedo, O.; Jochmans, D.; Leyssen, P.; Wang, K.; Neyts, J., et al. Kobophenol A Inhibits Binding of Host ACE2 Receptor with Spike RBD Domain of SARS-CoV-2, a Lead Compound for Blocking COVID-19. The journal of physical chemistry letters 2021, 12, 1793-1802.

(51) Williams-Noonan, B. J.; Todorova, N.; Kulkarni, K.; Aguilar, M.-I.; Yarovsky, I. An Active Site Inhibitor Induces Conformational Penalties for ACE2 Recognition by the Spike Protein of SARS-CoV-2. The Journal of Physical Chemistry B 2021,

(52) Anandakrishnan, R.; Aguilar, B.; Onufriev, A. V. H++ 3.0: automating p K prediction and the preparation of biomolecular structures for atomistic molecular modeling and simulations. Nucleic acids research 2012, 40, W537-W541.

(53) Sterling, T.; Irwin, J. J. ZINC 15-ligand discovery for everyone. Journal of chemical information and modeling 2015, 55, 2324-2337.

(54) O’Boyle, N. M.; Banck, M.; James, C. A.; Morley, C.; Vandermeersch, T.; Hutchison, G. R. Open Babel: An open chemical toolbox. Journal of cheminformatics 2011, 3, 33 .

(55) Trott, O.; Olson, A. J. Improving the speed and accuracy of docking with a new scoring 
function, efficient optimization, and multithreading. J. Comput. Chem 2010, 31, 455461.

(56) Skalic, M.; Martínez-Rosell, G.; Jiménez, J.; De Fabritiis, G. PlayMolecule BindScope: large scale CNN-based virtual screening on the web. Bioinformatics 2019, 35, 12371238.

(57) Jiménez, J.; Skalic, M.; Martinez-Rosell, G.; De Fabritiis, G. K deep: protein-ligand absolute binding affinity prediction via 3d-convolutional neural networks. Journal of chemical information and modeling 2018, 58, 287-296.

(58) Phillips, J. C.; Braun, R.; Wang, W.; Gumbart, J.; Tajkhorshid, E.; Villa, E.; Chipot, C.; Skeel, R. D.; Kale, L.; Schulten, K. Scalable molecular dynamics with NAMD. Journal of computational chemistry 2005, 26, 1781-1802.

(59) Jorgensen, W. L.; Chandrasekhar, J.; Madura, J. D.; Impey, R. W.; Klein, M. L. Comparison of simple potential functions for simulating liquid water. The Journal of chemical physics 1983, 79, 926-935.

(60) Yu, W.; He, X.; Vanommeslaeghe, K.; MacKerell Jr, A. D. Extension of the CHARMM general force field to sulfonyl-containing compounds and its utility in biomolecular simulations. Journal of computational chemistry 2012, 33, 2451-2468.

(61) Best, R. B.; Zhu, X.; Shim, J.; Lopes, P. E.; Mittal, J.; Feig, M.; MacKerell Jr, A. D. Optimization of the additive CHARMM all-atom protein force field targeting improved sampling of the backbone $\phi, \psi$ and side-chain $\chi 1$ and $\chi^{2}$ dihedral angles. Journal of chemical theory and computation 2012, 8, 3257-3273.

(62) Nosé, S.; Klein, M. Constant pressure molecular dynamics for molecular systems. Molecular Physics 1983, 50, 1055-1076. 
(63) Nosé, S. A molecular dynamics method for simulations in the canonical ensemble. Molecular physics 1984, 52, 255-268.

(64) Grest, G. S.; Kremer, K. Molecular dynamics simulation for polymers in the presence of a heat bath. Physical Review A 1986, 33, 3628.

(65) Essmann, U.; Perera, L.; Berkowitz, M. L.; Darden, T.; Lee, H.; Pedersen, L. G. A smooth particle mesh Ewald method. The Journal of chemical physics 1995, 103, 85778593.

(66) Ryckaert, J.-P.; Ciccotti, G.; Berendsen, H. J. Numerical integration of the cartesian equations of motion of a system with constraints: molecular dynamics of n-alkanes. Journal of computational physics 1977, 23, 327-341.

(67) Fu, H.; Cai, W.; Hénin, J.; Roux, B.; Chipot, C. New coarse variables for the accurate determination of standard binding free energies. Journal of chemical theory and computation 2017, 13, 5173-5178.

(68) Fu, H.; Gumbart, J. C.; Chen, H.; Shao, X.; Cai, W.; Chipot, C. BFEE: A userfriendly graphical interface facilitating absolute binding free-energy calculations. Journal of chemical information and modeling 2018, 58, 556-560.

(69) Gumbart, J. C.; Roux, B.; Chipot, C. Standard binding free energies from computer simulations: What is the best strategy? Journal of chemical theory and computation 2013, 9, 794-802.

(70) Fiorin, G.; Klein, M. L.; Hénin, J. Using collective variables to drive molecular dynamics simulations. Molecular Physics 2013, 111, 3345-3362.

(71) Phillips, J. C.; Hardy, D. J.; Maia, J. D.; Stone, J. E.; Ribeiro, J. V.; Bernardi, R. C.; Buch, R.; Fiorin, G.; Hénin, J.; Jiang, W., et al. Scalable molecular dynamics on 
CPU and GPU architectures with NAMD. The Journal of chemical physics 2020, 153, 044130.

(72) Fu, H.; Shao, X.; Chipot, C.; Cai, W. Extended adaptive biasing force algorithm. An on-the-fly implementation for accurate free-energy calculations. Journal of chemical theory and computation 2016, 12, 3506-3513.

(73) Lesage, A.; Lelievre, T.; Stoltz, G.; Hénin, J. Smoothed biasing forces yield unbiased free energies with the extended-system adaptive biasing force method. The Journal of Physical Chemistry B 2017, 121, 3676-3685.

(74) Nguyen, H. L.; Lan, P. D.; Thai, N. Q.; Nissley, D. A.; O’Brien, E. P.; Li, M. S. Does SARS-CoV-2 bind to human ACE2 more strongly than does SARS-CoV? The Journal of Physical Chemistry B 2020, 124, 7336-7347.

(75) Taka, E.; Yilmaz, S. Z.; Golcuk, M.; Kilinc, C.; Aktas, U.; Yildiz, A.; Gur, M. Critical Interactions Between the SARS-CoV-2 Spike Glycoprotein and the Human ACE2 Receptor. bioRxiv 2020,

(76) Vuong, Q. V.; Nguyen, T. T.; Li, M. S. A new method for navigating optimal direction for pulling ligand from binding pocket: application to ranking binding affinity by steered molecular dynamics. Journal of chemical information and modeling 2015, 55, 27312738.

(77) Laurini, E.; Marson, D.; Aulic, S.; Fermeglia, M.; Pricl, S. Computational alanine scanning and structural analysis of the SARS-CoV-2 spike protein/angiotensin-converting enzyme 2 complex. ACS nano 2020, 14, 11821-11830.

(78) Wang, Y.; Liu, M.; Gao, J. Enhanced receptor binding of SARS-CoV-2 through networks of hydrogen-bonding and hydrophobic interactions. Proceedings of the National Academy of Sciences 2020, 117, 13967-13974. 


\section{Graphical TOC Entry}

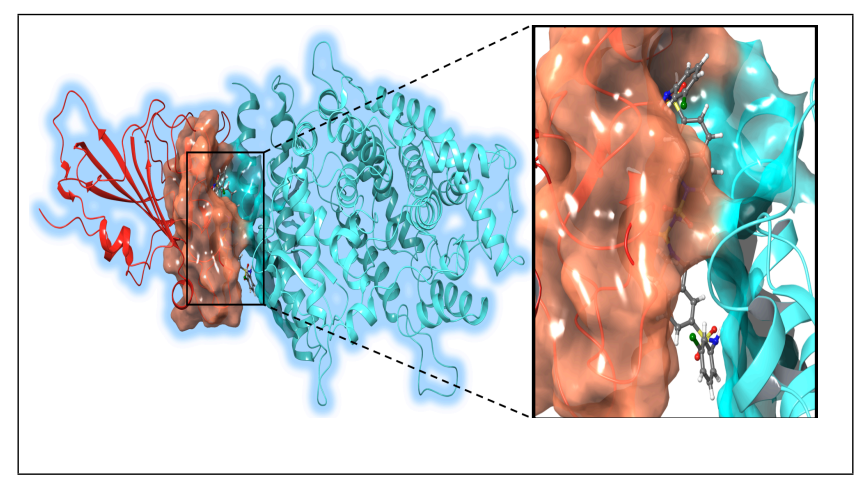

